\title{
Winterharte Gewürzstrauchgewächse (Calycanthaceae)
}

\author{
Veit Martin Dörken \& Annette Höggemeier
}

\begin{abstract}
Calycanthaceae are a small plant family of mostly deciduous species with aromatic roots, bark and leaves. Some of their floral traits have been interpreted as primitive. The paper focusses on species that are hardy enough for outdoor cultivation.
\end{abstract}

\section{Zusammenfassung}

Die Gewürzstrauchgewächse sind eine kleine Familie aus überwiegend laubabwerfenden Gehölzen mit aromatisch duftenden Wurzeln, Rinden und Blättern. Manche Merkmale ihrer Blüten werden als ursprünglich innerhalb der Angiospermen interpretiert. Nachfolgend werden die Gattungen vorgestellt, deren Arten in Deutschland ganzjährig im Freien kultiviert werden können.

\section{Kurzcharakteristik der Familie}

Vertreter der Calycanthaceae sind Holzgewächse mit stark aromatisch duftenden Wurzeln, Rinden und Blättern. Ihre zwittrigen, radiärsymmetrischen Blüten zeigen Merkmale, die als ursprünglich interpretiert werden. So gehen die Kelch- und Kronblätter übergangslos ineinander über, die vielen Staubblätter stehen spiralig. Die zahlreichen freien, nicht verwachsenen Fruchtblätter sind tief in das Gewebe der krug- förmigen Blütenachse eingesenkt. In mitteleuropäischen Gärten sind gelegentlich der Gewürzstrauch (Calycanthus) sowie die Winterblüte (Chimonanthus) anzutreffen.

Die Familie unfasst drei Gattungen (Mabberley 2008). Der Chinesische Gewürzstrauch, der erst in den 1980er Jahren nach Mitteleuropa gelangte, wurde zunächst zur Gattung Calycanthus gestellt, neuerdings je-

Abb. 1: Calycanthus floridus, blühend.

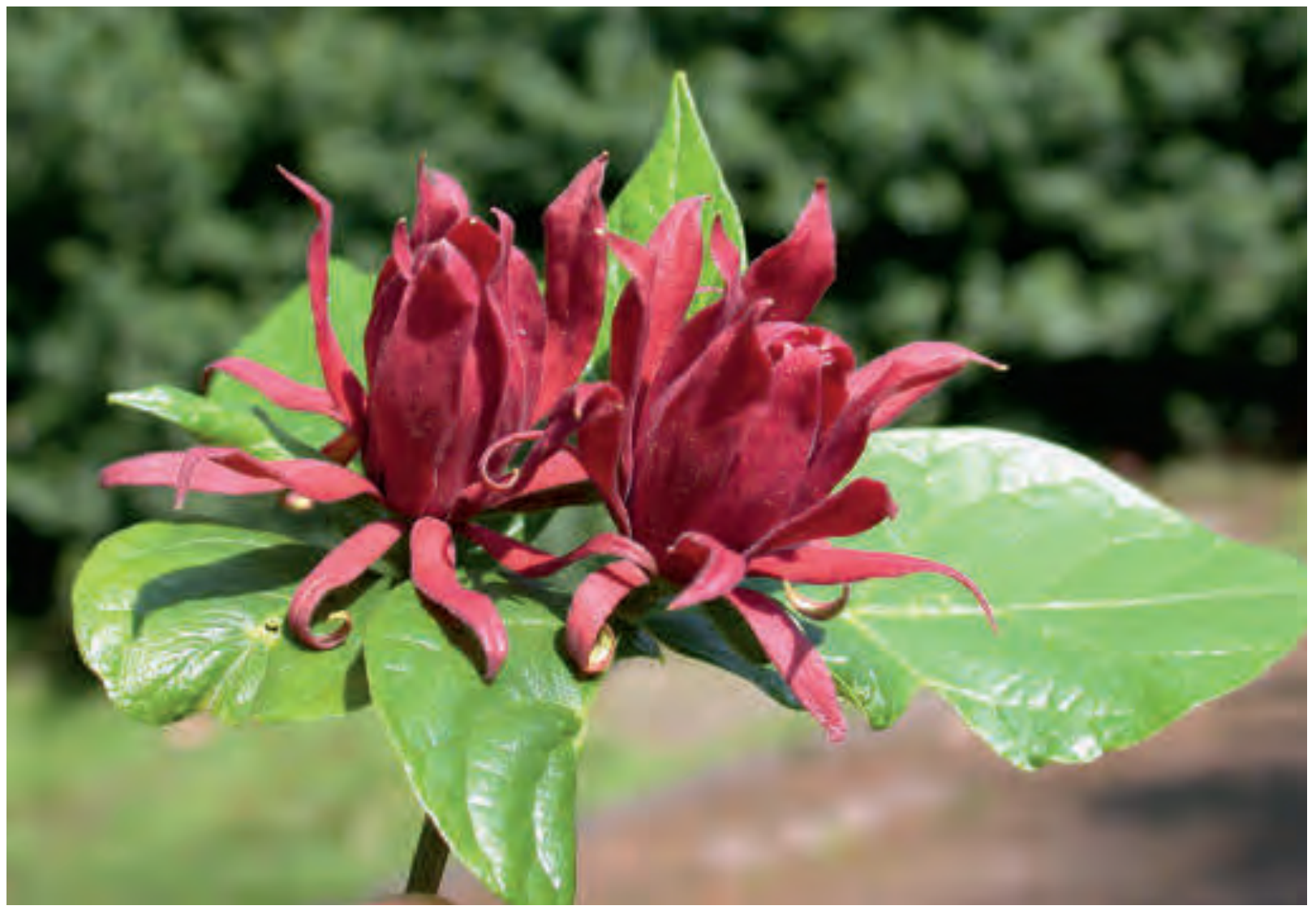




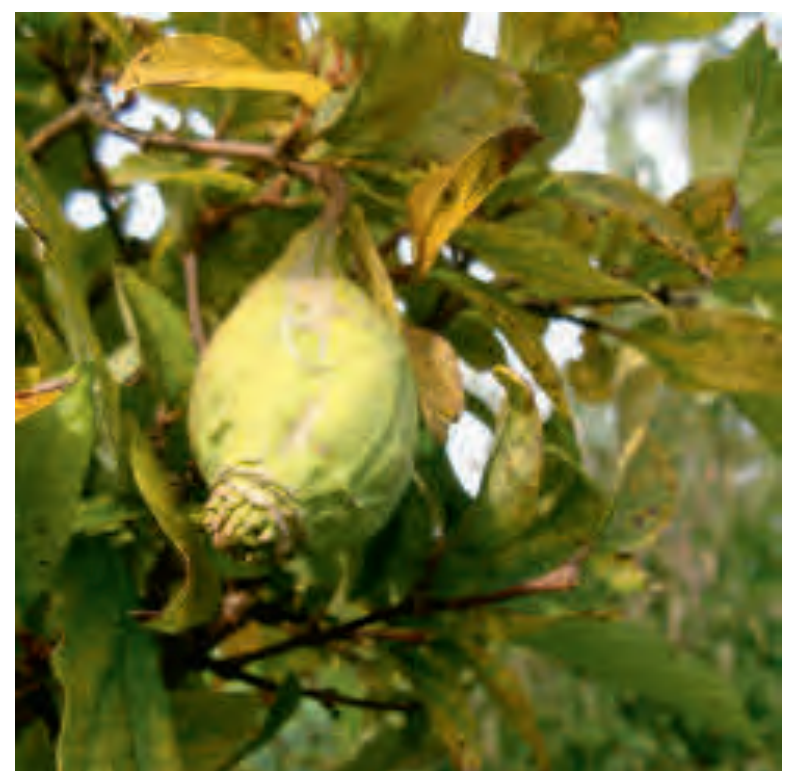

doch in die eigene monotypische Gattung Sinocalycanthus ausgegliedert.

Die namensgebende Gattung für die Familie ist Calycanthus (Gewürzstrauch). Der Gattungsname leitet sich von den griechischen Vokabeln kalyx (Kelch) und anthos (Blüte) ab. Der Name bezieht sich darauf, dass die Blütenblätter nicht in Kelch und Krone differenziert sind. Die deutsche Bezeichnung Gewürzstrauch greift den aromatischen Geruch auf.

\section{Calycanthus-Gewürzstrauch}

In Kalifornien und den südöstlichen Staaten der USA sind die amerikanischen Gewürzsträucher mit vier Arten heimisch. EveretT (1981) beklagt sich darüber, dass in Nordamerika die in den Gärten des 19. Jh. beliebte Gattung mittlerweile aus der Mode gekommen sei. Ursache dafür ist die zunehmende Popularität von Ziergehölzen aus Asien, die den modernen Vorlieben für auffallende Blüten und Düfte eher entgegenkommen. Der bekannteste und winterhärteste Vertreter ist der aus den östlichen USA stammende Strauch Calycanthus floridus. In Deutschland ist er nur an geschützten Standorten auf nährstoffreichen, gut drainierten Böden in sonnigen bis halbschattigen Lagen winterhart.

Es handelt sich um ein sommergrünes Gehölz bis $3 \mathrm{~m}$ Höhe. Triebe und Blätter, besonders im getrockneten Zustand, duften aufgrund

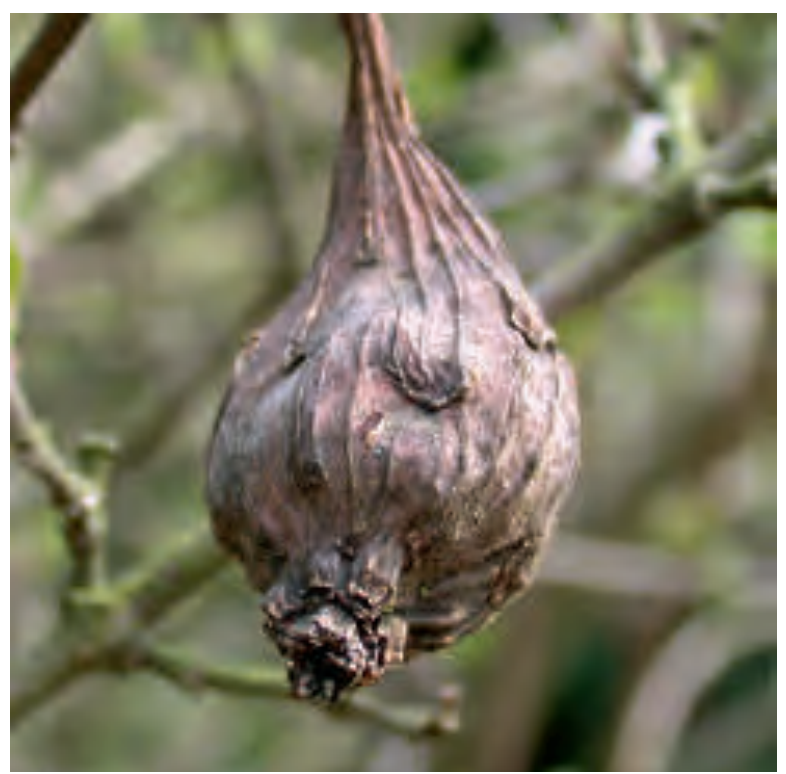

ihres hohen Gehaltes an ätherischen Ölen stark nach Nelken und Pfeffer. Drauf bezieht sich ein weiterer deutscher Name (Nelkenpfeffer), der nicht mit Piment (ebenso Nelkenpfeffer genannt) verwechselt werden sollte. Junge Triebe sind filzig behaart. Die gegenständigen, länglich-eiförmigen Blätter sind oberseits dunkelgrün und kahl, unterseits heller und dicht filzig behaart. Die dunkelroten bis zimtfarbenen und vor allem abends angenehm duftenden Blüten erscheinen von Juni bis Juli nach dem Laubaustrieb. Staub- und Fruchtblätter stehen an der Innenseite einer becherförmigen Blütenachse. Die inneren Staubblätter sind steril. Fertile und sterile Staubblätter entwickeln Futterkörperchen als Belohnung für die bestäubenden Käfer (B̈̈rtels 2001). Die mit Schuppenblättern besetzte Blütenachse ist zur Mündung hin verengt und zum Zeitpunkt der Samenreife fast ganz geschlossen. Die Früchte sind einsamige Nüsschen, die später vom zunächst fleischigen, später trockenen Blütenbecher vollständig umgeben werden und eine kugelige Ausbreitungseinheit bilden. Diese ist bei Calycanthus floridus im Gegensatz zu C. occidentalis zur Öffnung

Abb. 2 (links): Unreife Frucht von Calycanthus floridus.

Abb. 3 (rechts): Reife, eingetrocknete Frucht von Calycanthus floridus. 
hin fast ganz verschlossen. Die Wand des Fruchtbechers ist derb ledrig und behaart. Alle Teile des Echten Gewürzstrauches sind giftig, besonders die Samen. Sie enthalten das Alkaloid Calycanthin, das eine ähnliche Wirkung zeigt wie Strychnin. Aus dem natürlichen Verbreitungsgebiet der Art sind Berichte von vergifteten Weidetieren bekannt, die die Früchte gefressen hatten. Calycanthus floridus diente nordamerikanischen Ureinwohnern als Gewürz. Die Borke wurde in geringer Dosierung ähnlich wie Zimtrinde verwendet.

\section{Chimonanthus - Winterblüte}

Zur Gattung Chimonanthus werden sechs Arten gestellt. Ein gelegentlich in Sammlungen und Gärten zu findender Zierstrauch aus dieser Gattung ist die Winterblüte (Chimonathus praecox), ein sommergrüner Strauch aus China. Die botanische Gattungsbezeichnung Chimonanthus bezieht sich auf die frühe Blütezeit (cheimon = Winter, anthos = Blüte).

Die Winterblüte wird 2,5 $\mathrm{m}$ hoch. Junge Triebe sind anfänglich gräulich grün, später braun. Die bis $20 \mathrm{~cm}$ langen, elliptisch-eiförmigen Blätter stehen gegenständig. Der Blattrand ist ganzrandig bis leicht gebuchtet und durch kurze Borsten rau. Die 2,5 cm breiten Blüten erscheinen reichlich im zeitigen Winter von Dezember bis in den März hinein und verströmen einen intensiven Vanilleduft. Die äußeren Blütenhüllblätter sind kräftig gelb gefärbt, die inneren gelbbraun bis purpurfarben. Idealerweise in Hausnähe, am besten kontrastierend vor dunklem Hintergrund gepflanzt, erfreuen diese Sträucher mehrere Wochen lang mit ihren Blüten und deren Duft. Die Nussfrüchte werden maximal $3 \mathrm{~cm}$ lang. Sie sind von der länglich bis krugförmigen, 4 bis $8 \mathrm{~cm}$ langen Blütenachse umgeben und mehr oder weniger eingeschnürt. Die Fruchtstände bleiben meist den ganzen Winter über an den

Abb. 4 (oben): Göffnete Frucht mit Samen von Calycanthus floridus.

Abb. 5 (unten): Im Winter bei Schnee blühender Chimonanthus praecox.
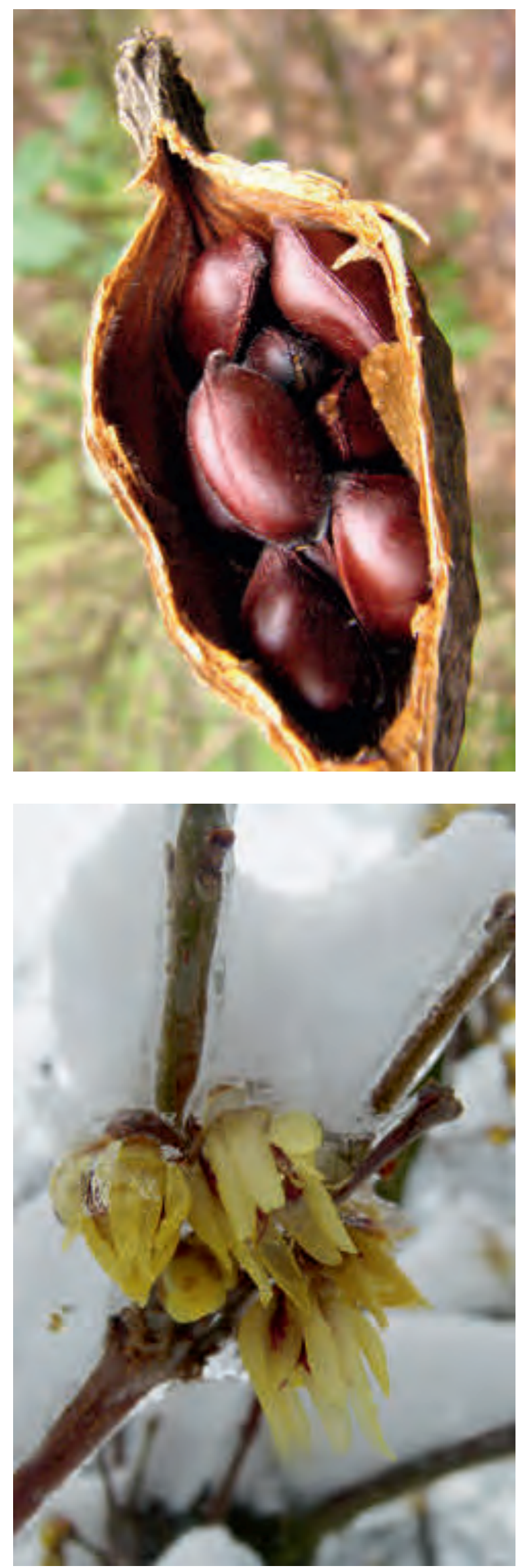


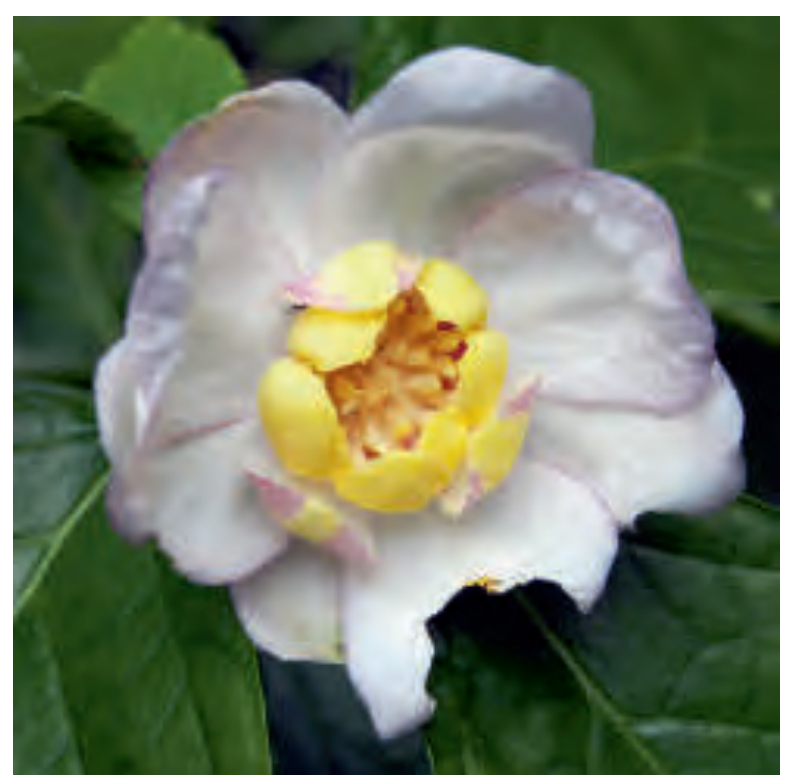

Pflanzen erhalten. Auch Chimonanthus enthält giftige Alkaloide. Winterblüte enthält in den Blättern neben Chimonantin auch Scylitt. Blütenextrakte werden in der Parfümindustrie genutzt.

Chimonanthus praecox ist an geschützten Standorten auch in Deutschland ausreichend frosthart. An den Boden stellt das Gehölz keine spezifischen Ansprüche, jeder Gartenboden wird vertragen, bevorzugt werden jedoch Kalkböden. Zu groß gewordene Individuen können problemlos zurückgeschnitten werden. Der Rückschnitt sollte jedoch unmittelbar nach der Blüte erfolgen, da der Strauch nur am zweijährigen Holz blüht.

\section{Sinocalycanthus chinensis - \\ Chinesischer Gewürzstrauch}

Der Chinesische Gewürzstrauch (Sinocalycanthus chinensis) ist die einzige Art der Gattung und stammt aus montanen Lagen Ost-Chinas. Dieser meist mehrstämmige, aufrecht wachsende Strauch ist nahe mit Calycanthus verwandt. Im Alter wird er unter hiesigen Klimabedingungen meist breiter als hoch, jedoch kaum über $2 \mathrm{~m}$ hoch. Seine Zweige hängen leicht über. Die Triebe sind mit zahlreichen auffälligen Korkwarzen (Lentizellen) besetzt. Im Austrieb sind die Blätter bronzefarben bis rötlich. Die breit bis länglich elliptischen Blätter werden $15 \mathrm{~cm}$ lang und sind zugespitzt. Der Blatt- rand ist gezähnt und leicht gewellt. Im Herbst färben sie sich kräftig goldgelb. Im Frühsommer erscheinen am Ende der Triebe die großen $10 \mathrm{~cm}$ breiten schalenförmigen Blüten. Die Blütenhülle ist nicht in Kelch- und Kronblätter gegliedert. Die Blütenblätter stehen in 2 Kreisen zu je 10 und die äußeren Blütenblätter sind fast doppelt so lang wie die inneren, rein weiß und am Rande leicht rosafarben überlaufen. Die inneren Blütenblätter sind etwas fleischig und zur Spitze hin gelblich, zur Basis hin weiß gefärbt mit dunkelroten Flecken. Jede Blüte enthält bis 25 gelbe Staubblätter. Die bei der Reife birnenförmige, $3,5 \mathrm{~cm}$ dicke, feigenartige Sammelnussfrucht ist dunkelbraun gefärbt. Da der Chinesische Gewürzstrauch besonders in der Jugend empfindlich auf allzu tiefe Wintertemperaturen reagiert, ist bei der Pflanzung auf einen geschützten Standort zu achten sowie zumindest in der Jugendphase auf geeigneten Winterschutz. Im Botanischen Garten Bochum hat das dort im Freiland ausgepflanzte Individuum im Schutze einiger größerer Gehölze auch die Extremwinter 2008/2009 sowie 2009/2010 mit Temperaturminima von $-20^{\circ} \mathrm{C}$ schadlos überstanden.

Verschiedene Calycanthaceae gedeihen auch im Palmengartenn, darunter Chinesischer Gewürzstrauch, Chimonanthus praecox und C. nitens sowie Calycanthus floridus var. glaucus.

\section{Literatur \\ Mabberley, D. J. 2008: Mabberley's Plant Book. 3 Aufl. - Cambridge. \\ Everett, T. H. 1981: The New York Botanical Garden illustrated encyclopedia of horticulture. - New York \& London.}

Abb. 6: Sinocalycanthus chinensis. 